\title{
Flexible metabolism in Metarhizium anisopliae and Beauveria bassiana: role of the glyoxylate cycle during insect pathogenesis
}

\begin{abstract}
Correspondence
Michael J. Bidochka

bidochka@brocku.ca
\end{abstract}

Received 17 June 2010

Revised 20 September 2010

Accepted 5 October 2010

\author{
Israel Enrique Padilla-Guerrero, ${ }^{1,2}$ Larissa Barelli, ${ }^{2}$ \\ Gloria Angélica González-Hernández, ${ }^{1}$ Juan Carlos Torres-Guzmán ${ }^{1}$ \\ and Michael J. Bidochka² \\ ${ }^{1}$ Laboratorio de Genética Molecular de Hongos, Departamento de Biología, Universidad de \\ Guanajuato, Noria Alta s/n, Guanajuato, Gto., Mexico \\ ${ }^{2}$ Department of Biological Sciences, Brock University, St Catharines, ON L2S 3A1, Canada
}

\section{INTRODUCTION}

The entomopathogenic fungi Metarhizium anisopliae and Beauveria bassiana encounter different niches within the host during the course of insect infection. These niches are highly variable with respect to the types and abundance of available nutrients. First, the fungal conidia adhere to the surface of the insect cuticle. This is followed by germination, production of an appressorium and hyphal penetration through the cuticle where the primary nutrients are bound as protein and chitin co-polymers in the cuticle matrix. Once in the nutrient-rich insect haemolymph, which contains accessible sugars, proteins and lipids, the fungi differentiate into blastospores and contend with phagocytic haemocytes. The fungus grows within the haemolymph and eventually hyphae re-emerge from the insect cadaver and conidiate on the surface of the cadaver. During each stage of pathogenesis, a specific

Abbreviations: 3-NP, 3-nitropropionate; ICL, isocitrate lyase.

A supplementary figure showing the upregulation of the Ma-ic/ promoter is available with the online version of this paper. subset of virulence genes is upregulated (Clarkson \& Charnley, 1996; Small \& Bidochka, 2005; Thomas \& Read, 2007; Wang \& St Leger, 2006, 2007; Wang et al., 2008).

During the course of insect infection, M. anisopliae and $B$. bassiana encounter different carbon sources; proteins, hydrocarbons, fatty acids and lipids may be found on the surfaces of insect cuticle, the fat body, haemolymph and within insect haemocytes (Arrese \& Soulages, 2010; Jarrold et al., 2007; Prasad et al., 1986). In other fungi, utilization of non-fermentable carbon sources activates the upregulation of the glyoxylate cycle for cell metabolism and biosynthesis (Lorenz \& Fink, 2001). The glyoxylate cycle is a modified shunt of the tricarboxylic acid cycle, the result of which is the production of malate and succinate from two molecules of acetyl-CoA derived from the metabolism of acetate or from the degradation of ethanol, fatty acids or poly-b-hydroxybutyrate (Dunn et al., 2009). Several plant and human pathogenic fungi and bacteria utilise the glyoxylate cycle during host infection (Dunn et al., 2009).

Here, we monitored the activity of the central carbon metabolic pathway of the glyoxylate cycle, in particular 
isocitrate lyase (ICL), a key enzyme in the glyoxylate cycle in $M$. anisopliae and B. bassiana, under various growth conditions. We also monitored the upregulation of the glyoxylate cycle during appressorium formation and insect infection by $M$. anisopliae. ICL has been implicated in survival of the human pathogenic fungus Candida albicans within mammalian macrophages (Lorenz \& Fink, 2001). We constructed a reporter gene (mCherry fluorescent protein or green fluorescent protein) under the control of the $i c l$ promoter in M. anisopliae in order to investigate the upregulation of the glyoxylate cycle in M. anisopliae during phagocytosis by insect haemocytes.

\section{METHODS}

Fungal and bacterial strains. M. anisopliae strain ARSEF 2575 and B. bassiana strain ARSEF 252 were obtained from the United States Department of Agriculture Collection of Entomopathogenic Fungal Cultures, Ithaca, NY, USA. Cultures were grown on potato dextrose agar (PDA) at $27{ }^{\circ} \mathrm{C}$ for 10 days. Escherichia coli JM109 was used for DNA ligations and transformations.

ICL assay. M. anisopliae and B. bassiana conidia were inoculated into yeast extract-peptone-glucose (YPD) broth cultures, grown for $72 \mathrm{~h}$, $27{ }^{\circ} \mathrm{C}$ at 180 r.p.m. The mycelia were harvested by vacuum filtration onto Whatman no. 2 filter paper, washed with sterile distilled water, and transferred into $0.025 \%$ yeast extract (YE) broth for $24 \mathrm{~h}$ followed by another water wash. Previous experimentation determined that this 'starvation phase' in a low nutrient medium $(0.025 \%$ YE) was necessary to deplete fungal hyphae of carbohydrate reserves that obfuscated results if fungi were transferred directly into media containing various carbon sources from the nutrient-rich YPD broth. To determine the inductive effects under various growth conditions, approximately $0.2 \mathrm{~g}$ (wet weight) of washed mycelia were inoculated into $50 \mathrm{ml}$ distilled, deionized water containing only the carbon source $2 \%$ glucose, $2 \%$ acetate, $2 \%$ raffinose, $3 \%$ ethanol, $0.05 \%$ oleic acid or $2 \%$ glucose $-2 \%$ acetate and grown for $6 \mathrm{~h}$. The mycelia were again harvested by vacuum filtration onto Whatman no. 2 filter paper and stored at $-80{ }^{\circ} \mathrm{C}$.

We monitored ICL activity using an enzymic assay during germination of M. anisopliae and B. bassiana. Conidia $\left(1 \times 10^{7}\right.$ conidia $\left.\mathrm{ml}^{-1}\right)$ were inoculated into $100 \mathrm{ml}$ minimal medium (M-100) supplemented with $1 \mathrm{~g}$ glucose, $0.3 \mathrm{~g} \mathrm{KNO}_{3}$ and $6 \mathrm{ml} \mathrm{M}-100$ salt solution and grown at $27^{\circ} \mathrm{C}$ and 180 r.p.m. At 3, 6, 12, 18 and 24 h post-inoculation, the conidia were re-collected by centrifugation $(6000 \mathrm{~g}$ for $5 \mathrm{~min}$ ) and stored at $-80{ }^{\circ} \mathrm{C}$. The conidial sample was ground using a sterile mortar and pestle in liquid nitrogen. The finely ground sample was suspended and vortexed in $300 \mu \mathrm{l}$ extraction buffer composed of $21.8 \mathrm{mM}$ HEPES, $1.0 \mathrm{mM}$ dithiothreitol, $1.0 \mathrm{mM} \mathrm{KCl}, 0.125 \%$ Triton X-100 and mini EDTA-free protease inhibitor cocktail tablet (Roche). ICL activity was detected using the following final assay concentration: $25 \mathrm{mM}$ imidazole buffer ( $\mathrm{pH} 6.8$ ), $5 \mathrm{mM} \mathrm{MgCl}_{2}, 4 \mathrm{mM}$ phenylhydrazine and $1 \mathrm{mM}$ isocitric acid. Enzymic assays were carried out in 96-well plates. Each well contained $200 \mu \mathrm{l}$ reaction master mix and $50 \mu \mathrm{l}$ protein extract. The absorbance was taken at $324 \mathrm{~nm}$ at $30{ }^{\circ} \mathrm{C}$ using a temperature-controlled KC4 96-well plate reader (Bio-Tek). ICL specific activity was reported as $10^{-5} \times \mu$ mol phenylhydrazine glyoxylate generated $\min ^{-1}$ (mg protein $)^{-1}$.

Gene cloning. Fungal genomic DNA was isolated using the plant/ fungus DNA isolation kit (Norgen). A fragment of the genes encoding citrate synthase-1 (Ma-cit1), malate synthase and ICL ( $M a-i c l)$ from $M$. anisopliae was cloned using degenerate hybrid primers constructed with the program iCODEHOP v1 (Rose et al., 2003) and identified using BLASTX (Table 1). We also found another citrate synthase gene (Ma-cit2) while gene-walking from the $5^{\prime}$ end of $M a$-icl. The full-length $\mathrm{Ma}$-icl and $\mathrm{Ma}$-cit2 genes were subsequently cloned by PCR walking using the Y-shaped adaptor dependent extension (YADE) method and SiteFinding-PCR (Fang et al., 2007; Tan et al., 2005). We also cloned a 2206 bp DNA fragment, which contained the partial sequence for $\mathrm{Bb}$-icl, using an EST identified in previous work (Cho et al., 2006a, b) by using the SiteFinding-PCR method. PCR was performed using JumpStart Taq DNA polymerase (Sigma).

RT-PCR. RT-PCR was used to examine transcript levels of genes representative of the glyoxylate and TCA cycles. Primers for RT-PCR were designed for isocitrate dehydrogenase (idh), aconitase (aco), citrate synthase $(c i t)$, malate synthase $(\mathrm{mls})$ and ICL $(i c l)$, and were identified in this study ( $M$. anisopliae) or from an EST analysis of B. bassiana transcripts from previous work (Cho et al., 2006a, b). As the reference, the $18 \mathrm{~S}$ rRNA gene was utilized for the two fungi. The primers and GenBank accession numbers are listed in Table 1. M. anisopliae and B. bassiana conidia were inoculated into YPD broth cultures, grown for $72 \mathrm{~h}, 27{ }^{\circ} \mathrm{C}$ at 180 r.p.m. Growth conditions and mycelial recovery were identical to those of the enzymic assays except that fungi were also grown in Manduca sexta haemolymph in a $10 \mathrm{ml}$ polycarbonate tube without air space for 1, 3, 6, 9 and $12 \mathrm{~h}$. Fungi were also grown in $1 \% \mathrm{w} / \mathrm{v}$ locust cuticle, giant cockroach cuticle, Manduca sexta cuticle, chitin, ground tomato stem, cellulose, starch and bean root exudate (Wang \& St Leger, 2007). The RNA was extracted from ground mycelia using TRI-reagent (SigmaAldrich) and treated with RQ1 RNase-free DNase (Promega). Total RNA $(1 \mu \mathrm{g})$ was used for cDNA synthesis according to manufacturer's instructions using the DyNAmo cDNA kit (Finnzymes). The PCR

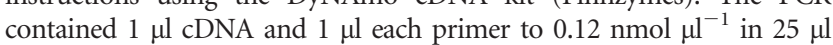
final volume with $0.5 \mu \mathrm{Taq}$ DNA polymerase and ThermoPol buffer (New England Biolabs). The conditions for amplification were $95{ }^{\circ} \mathrm{C}$ for $1 \mathrm{~min}$, followed by 30 cycles of $20 \mathrm{~s}$ denaturation at $95^{\circ} \mathrm{C}, 45 \mathrm{~s}$ annealing at $60{ }^{\circ} \mathrm{C}$ and $1 \mathrm{~min}$ polymerization at $72{ }^{\circ} \mathrm{C}$, followed by $72{ }^{\circ} \mathrm{C}$ for $3 \mathrm{~min}$. The RT-PCR was performed independently twice.

Construction of the fluorescent protein expression vector using the Ma-icl promoter. For construction of the pMa-Picl-gfp or $\mathrm{pMa}$-Picl-mCherry expression vector, a $907 \mathrm{bp} \mathrm{Ma}$-icl promoter fragment was amplified from DNA with the primers ICL-Promo (5'-TGGATCCGGTTGTCGATGTTGGGCT, BamHI restriction site underlined) and ICL-ProFP (5'-CGCCCTTGCTCACCATAGTGACGATGATGGCCAA). The coding sequence of the fluorescent proteins eGFP (Fang et al., 2006) and mCherry (Araujo-Palomares et al., 2009) was amplified with the primer pair FP-U (5'-ATGAGTAAAGGAGAAGAACTTT) and FP-L (5'-CTATTTGTATAGTTCATCCATG). The two fragments (promoter and fluorescent protein) were fused by PCR using the primers ICL-Promo and FP-L. The $1635 \mathrm{bp}$ and 1625 bp products for the fused pMa-Picl-gfp and pMa-Picl-mCherry, respectively, were subcloned into pGEM-T Easy (Promega). After sequence verification, the resulting plasmids were digested with BamHI and EcoRI and ligated into plasmid pBARGEM7-2. The circular plasmids pMa-Picl-gfp and pMa-Picl-mCherry were used to transform M. anisopliae (Wang \& St Leger, 2006). The transformants were selected in media containing $200 \mu \mathrm{g}$ glufosinate-ammonium $\mathrm{ml}^{-1}$ and screened for fluorescence. Integration of the construct into the genome was verified by PCR. Functionality of the promoter was evaluated by growing the transformants in $100 \mathrm{ml}$ minimal medium (M-100) supplemented with $1 \mathrm{~g}$ glucose or acetate, $0.3 \mathrm{~g} \mathrm{KNO}_{3}$ and $6 \mathrm{ml} \mathrm{M}-100$ salt solution.

Inhibition of ICL activity using 3-nitropropionate (3-NP). For inhibition of ICL activity we used 3-NP (Ebel et al., 2006; Kim et al., 2006) a carbanion form of 3-nitropropionic acid (Sigma) (Schloss \& Cleland, 1982). Minimal medium, M-100, was supplemented with glucose or acetate and $8 \mathrm{mM}$ or $6 \mathrm{mM}$ of 3-NP for M. anisopliae or $B$. 
Table 1. Oligonucleotides used in this study

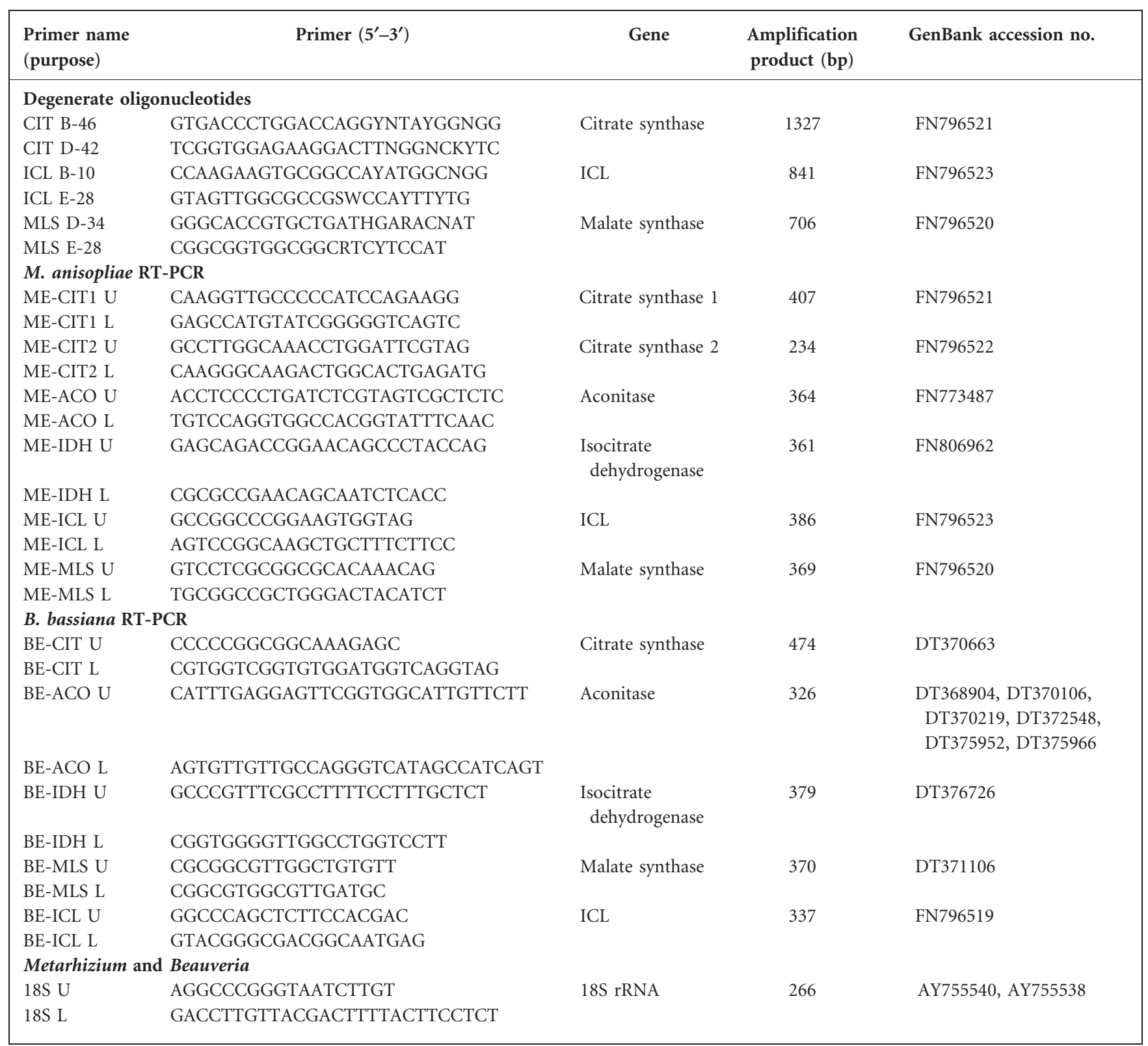

bassiana, respectively. We also observed the effect of $8 \mathrm{mM} 3-\mathrm{NP}$ on M. anisopliae appressorium formation in $0.1 \%$ alanine in a Petri dish.

Glyoxylate cycle during the infection process. Conidia $(20 \mu \mathrm{l}$ of

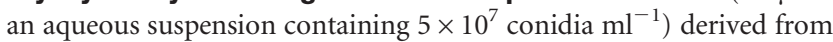
fungi expressing Ma-Picl-mCherry were injected into the first proleg of fifth-instar larvae of Manduca sexta. Haemolymph was then collected at $0,3,6$ and 9 h post-inoculation and haemocytes were concentrated by centrifugation at $150 \mathrm{~g}$ for $5 \mathrm{~min}$; the samples were observed microscopically. We also determined if $\mathrm{Ma}$-Picl-mCherry was expressed by $M$. anisopliae $48 \mathrm{~h}$ post-inoculation in Manduca sexta and during fungal re-emergence from the insect cadaver (5 days). We also determined if Ma-Picl-mCherry was expressed during appressorium formation when fungi were grown in $0.1 \%$ alanine in a Petri dish.

Microscopy. Imaging was performed using a Nikon OPTIPHOT-2 microscope equipped with epifluorescent illumination. Cells expressing
mCherry or eGFP were imaged using TRITC HYQ 530-550/565/690650 or GFP HYQ (LP) 425-475/480/485LP filter cubes (Chroma). The pictures for bright-field and fluorescence were obtained using a SPOTRT colour digital camera and the SPOT advanced software version 4.6. In all the microscopic studies, at least 200 cells were observed.

\section{RESULTS}

\section{Detection of ICL activity}

ICL and malate synthase are intermediate enzymes in the glyoxylate cycle involved in the metabolism of 2-carbon $\left(\mathrm{C}_{2}\right)$ compounds, such as acetate, and hydrolytic products of fatty acids. Although the glyoxylate cycle has been found in many fungi, we first determined whether at least one 
intermediate enzyme of the glyoxylate cycle, ICL, was present in M. anisopliae and B. bassiana. Enzymic assays determined that ICL activity was present in these fungi and that the expression of ICL differed in various media (Fig. 1). Generally, ICL enzymic activities in M. anisopliae and $B$. bassiana were greatest when grown in the presence of a $\mathrm{C}_{2}$ compound (acetate and ethanol) and were reduced when glucose and raffinose were used as sole carbon sources. The addition of glucose and acetate reduced ICL activity in $M$. anisopliae, suggesting catabolic repression, which was not observed in B. bassiana. Both fungi also showed ICL activity when grown in the presence of oleic acid.

\section{Isolation of genes in the central carbon metabolic pathway}

Once we showed the existence of ICL activity, we cloned fragments of the genes from M. anisopliae and B. bassiana involved in the glyoxylate cycle (ICL and malate synthase) as well as genes that code for enzymes in the TCA cycle (citrate synthase, aconitase and isocitrate dehydrogenase; Table 1), which occurred before or after the bifurcation of the central carbon pathway to the glyoxylate cycle. For $M$. anisopliae, we isolated a $4509 \mathrm{bp}$ DNA fragment that contained two genes, the ORF (1854 bp) for ICL ( $M a-i c l)$ and, fortuitously, a second gene encoding citrate synthase (Ma-cit2), which has two introns and an ORF of $1416 \mathrm{bp}$. The fragment also contained an intervening region between the ORFs of Ma-icl and Ma-cit2 of just over $907 \mathrm{bp}$, which coincidently contained a shared bidirectional promoter region (Fig. 2). Putative transcriptional binding elements found in this region include a CREA region, responsive to glucose repression (SYGGRG), homologous to that in Aspergillus (Cubero \& Scazzocchio, 1994), a nitrogen

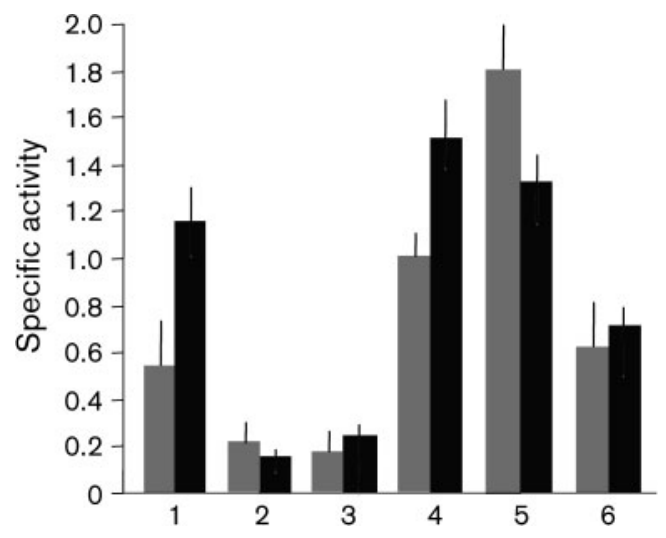

Fig. 1. ICL activities in M. anisopliae (grey) and B. bassiana (black) when grown on different carbon sources. 1, 2\% Glucose, $2 \%$ sodium acetate; 2, $2 \%$ glucose; 3, 2\% raffinose; 4, 3\% ethanol; $5,2 \%$ sodium acetate; $6,0.5 \%$ oleic acid. Specific activity is measured as $10^{-5} \times \mu \mathrm{mol}$ phenylhydrazine glyoxylate produced $\min ^{-1}$ (mg protein) $)^{-1}$. regulatory element, AREA (HGATAR) (Ravagnani et al., 1997), and an acetate regulatory element, FacB (TCC/G N8-10 C/GGA or GCA/C N8-10 T/GGC) homologous to that found in Aspergillus (Todd et al., 1998). The bidirectional icl-cit2 promoter of Metarhizium is rich in these elements (Fig. 2), particularly the acetate-responsive element, FacB.

\section{Phylogenetic analysis of $\mathbf{M a - i c l}$ and $\mathbf{B b}$-icl}

Fig. 3 shows the phylogenetic relationship of the $i c l$ genes from B. bassiana (Bb-icl) and M. anisopliae $(M a-i c l)$ as well as icl-like ORF genes from other ascomycete and basidiomycete fungi obtained using CLUSTAL_x. The $B b-i c l$ gene was most closely related to Chaetomium globosum in the ascomycete clade. Meanwhile $\mathrm{Ma}$-icl was most closely related to the icl gene from Trichoderma reesei in the basidiomycete clade.

\section{Gene expression}

M. anisopliae and B. bassiana were grown in various media to study the induction of the glyoxylate cycle (Fig. 4). RTPCR analyses show high expression of $i c l$ and $\mathrm{mls}$ in acetate or ethanol $\left(\mathrm{C}_{2}\right.$ compounds), and low expression in glucose or raffinose. These results support those observed in the enzymic assays. However, differences in the regulation of the other representative genes in the central carbon metabolic pathways between $M$. anisopliae and B. bassiana were observed. In $B$. bassiana, the presence of glucose with acetate did not repress gene expression of the glyoxylate cycle genes. In contrast with this, glyoxylate cycle genes in M. anisopliae were repressed in the presence of glucose with acetate (Fig. 4). These differences were also observed in the regulation of the glyoxylate cycle between $M$. anisopliae and $B$. bassiana, when grown in insect haemolymph. The expression and regulation of $\mathrm{Ma}$-icl and Ma-cit2 were identical in the presence of various carbon sources, possibly since they shared a bidirectional promoter (Fig. 4).

A time-course experiment to determine the expression of M. anisopliae and B. bassiana genes in the central metabolic pathway was performed when fungi were grown in insect (Manduca sexta) haemolymph in limiting oxygen for up to $12 \mathrm{~h}$ (Fig. 4). This shows that the $\mathrm{Ma}$-icl and $\mathrm{Ma}$-cit2 genes of $M$. anisopliae in haemolymph are upregulated and that Ma-cit1 is downregulated. In B. bassiana, the level of transcripts indicated that genes involved in the glyoxylate cycle were not induced when grown in the haemolymph.

M. anisopliae is a facultative insect pathogen that can also occupy non-insect host niches such as saprobic growth and the plant rhizosphere (Hu \& St Leger, 2002; St Leger, 2008). Patterns of regulation of $\mathrm{Ma}$-icl and $\mathrm{Ma}$-cit2 during growth on insect (locust, giant cockroach or Manduca sexta) cuticles and plant-derived compounds (bean root exudate, tomato stems, cellulose or starch) show that $\mathrm{Ma}$ $i c l$ and $M a-c i t 2$ have similar patterns of regulation (Fig. 5). 


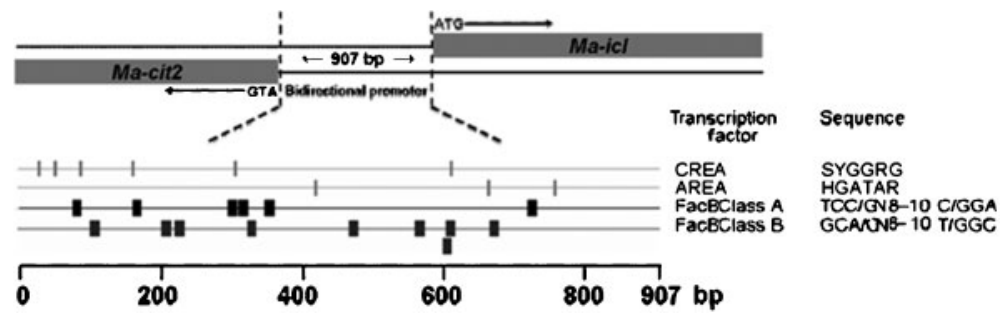

Fig. 2. Organization and potential core promoter elements for $\mathrm{Ma}$-icl and Ma-cit2. The diagram shows the intergenic region between the Ma-cit2 and Ma-icl genes. The ATG codons of both genes and their orientation are shown. Squares ( $\boldsymbol{\square})$ show the position and putative transcriptional binding elements involved in the regulation for glucose (CREA), acetate (FacB) and nitrogen (AREA).

\section{Determination of $\mathbf{M a - i c l}$ promoter activity}

The Ma-icl promoter and fluorescent protein fusions were constructed to monitor the in vivo regulation of the glyoxylate cycle of $M$. anisopliae in pathogenic and saprophytic conditions. First, the expression patterns of these promoter fusions were examined when transformants were grown in glucose or acetate. A greater level of fluorescence was observed when the fungi were grown in acetate, particularly at $48 \mathrm{~h}$, but not in glucose. Some fluorescence was observed in conidia, which suggests constitutive levels of $\mathrm{Ma}$-icl (Fig. 6). Enzymic
ICL assays in conidia and during conidial germination concurred with these results (Fig. 7a).

\section{Role of the glyoxylate cycle in the conidial germination and appressorium formation}

We monitored the enzymic activity of the glyoxylate cycle intermediate ICL during conidial germination in $M$. anisopliae and B. bassiana throughout the $24 \mathrm{~h}$ after germination in medium containing glucose. Glucose was

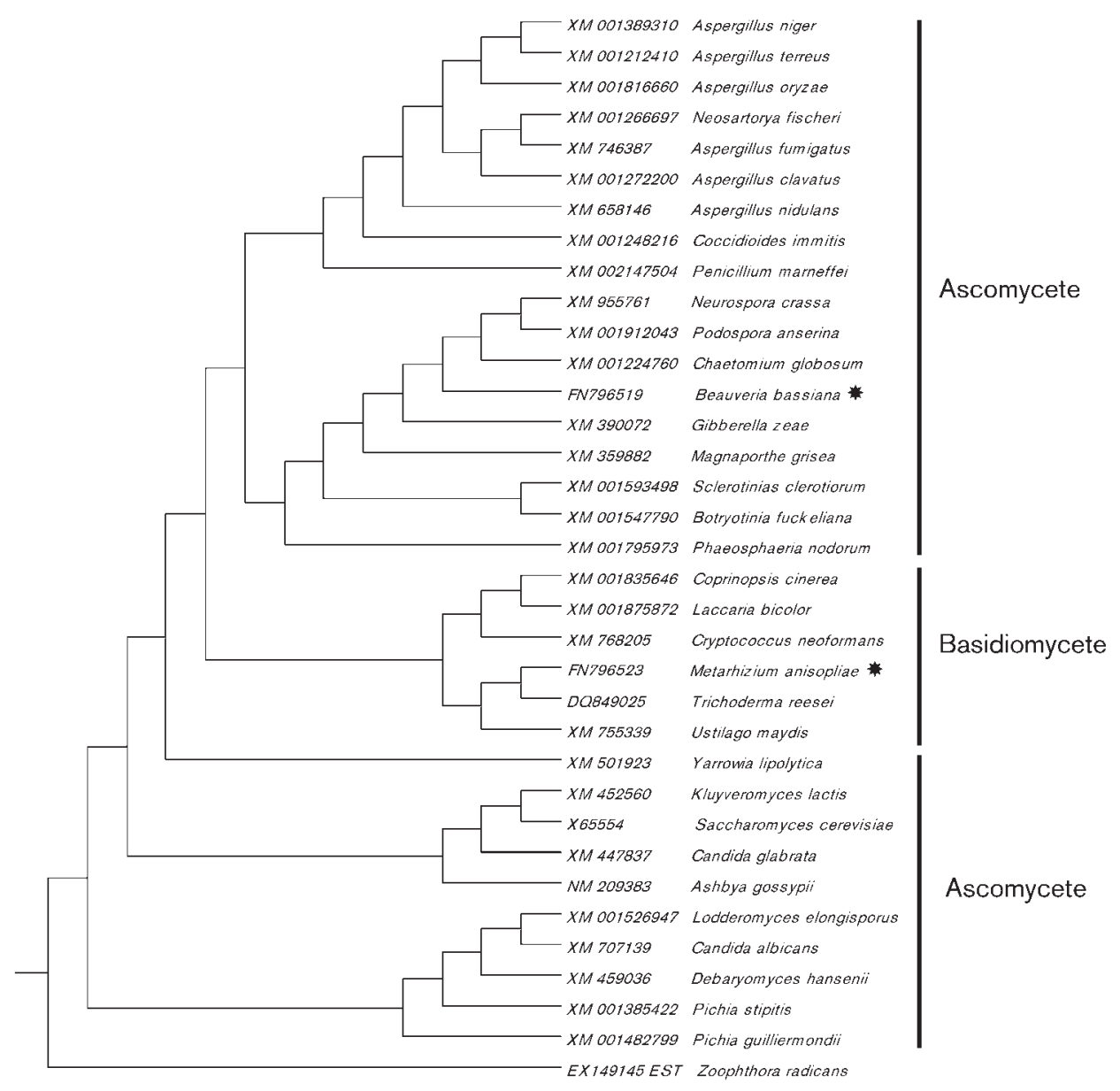

Fig. 3. Phylogenetic analysis of fungal ICL including $B$. bassiana and $M$. anisopliae (asterisks). Relationships were inferred by using the CLUSTAL_X method. The GenBank accession numbers of the sequences are shown alongside the names of the fungal species. 


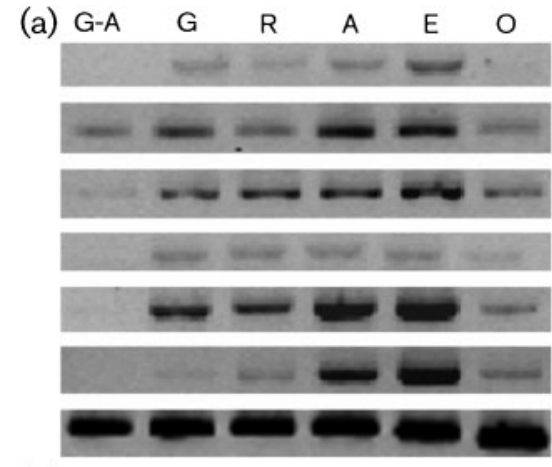

(b) G-A $\quad G \quad R \quad A \quad E \quad O$

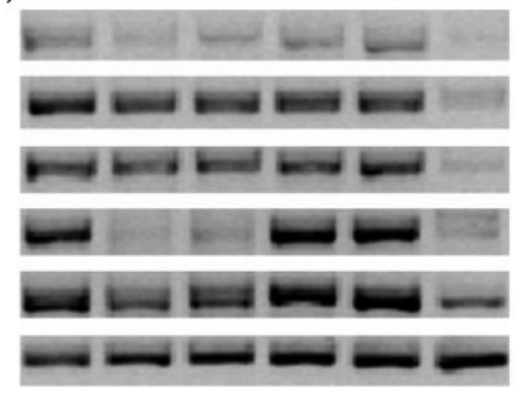

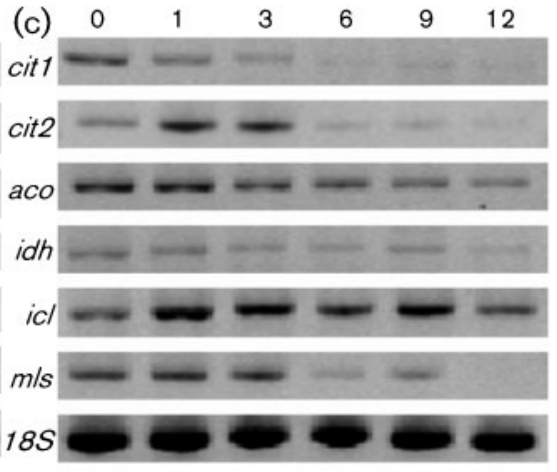

(d)

cit1

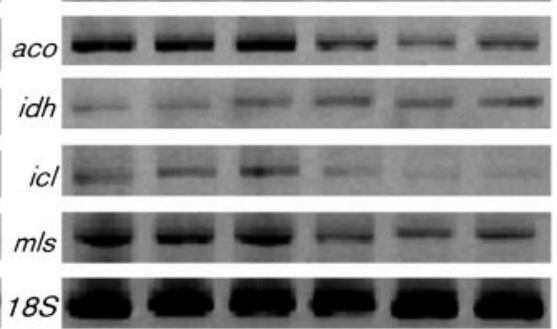

Fig. 4. RT-PCR analysis of the expression of genes representative of the central carbon metabolic pathway of $M$. anisopliae and $B$. bassiana grown with different carbon sources and in haemolymph. (a) M. anisopliae and (b) $B$. bassiana with various carbon sources after $6 \mathrm{~h}$ : $\mathrm{G}$, glucose; $\mathrm{R}$, raffinose; $A$, acetate; $E$, ethanol; O, oleic acid; G-A, glucose-acetate. (c) M. anisopliae and (d) B. bassiana in insect (Manduca sexta) haemolymph under low oxygen conditions up to $12 \mathrm{~h}$. cit, Citrate synthase; aco, aconitase; idh, isocitrate dehydrogenase; icl, ICL; $\mathrm{m} / \mathrm{s}$, malate synthase; 18S, 18sRNA control). Experiments were performed independently twice. utilized since it did not exogenously induce the glyoxylate cycle (see previous results) thus allowing the determination of constitutive levels of ICL. The experiment revealed the presence of ICL in germinating conidia of $M$. anisopliae and B. bassiana (Fig. 7a). A peak in ICL activity was observed at 9 and $18 \mathrm{~h}$ post-germination for M. anisopliae and B. bassiana, respectively.

First, we tested the concentrations of 3-NP, which resulted in $100 \%$ loss of germination (after a $24 \mathrm{~h}$ incubation) in acetate medium and determined that 8 and $6 \mathrm{mM} \mathrm{3-NP}$ for M. anisopliae and B. bassiana, respectively, could stimulate this loss. We then used these concentrations to monitor fungal germination in medium supplemented with glucose. We observed a delay in the rate of germination for $M$. anisopliae and B. bassiana conidia in glucose medium with 3-NP compared with the control (without 3-NP) after a $24 \mathrm{~h}$ incubation (Fig. $7 \mathrm{~b}$ ).

We also evaluated the effect of 3-NP on appressorium formation in M. anisopliae in a Petri dish containing $0.1 \%$ alanine (St Leger et al., 1989). Appressorium formation was inhibited in the presence of 3-NP (Fig. 7c). However, no differences were observed in conidial germination with or without 3-NP under these conditions (Fig. 7d).

\section{$M a-i c l$ in the infection process}

M. anisopliae can avoid insect haemocyte phagocytosis, perhaps originating as the ability to survive amoebic phagocytosis (Bidochka et al., 2010). We followed the expression of ICL in M. anisopliae during insect haemocytic phagocytosis using mCherry linked to the $\mathrm{Ma}$-icl promoter. We also used $g f p$ linked to the $M a-i c l$ promoter but observed less intense fluorescence than in Ma-Picl-mCherry (Supplementary Fig. S1). We observed upregulation of icl in $M$. anisopliae when it was phagocytosed by insect haemocytes (Fig. 8b-d), when the mycelium re-emerged in the insect cadaver (Fig. 8g) and in the formation of appressorium (Fig. $8 \mathrm{f}$ ), but not when the fungus was proliferating within the insect haemocoel (Fig. 8e).

\section{DISCUSSION}

We investigated the role of metabolism, particularly the glyoxylate cycle, in M. anisopliae and B. bassiana during the occupation of various niches, as insect pathogens, under saprobic conditions or during growth in plant-derived compounds. The glyoxylate cycle has previously been reported to play an important role in the virulence of fungi

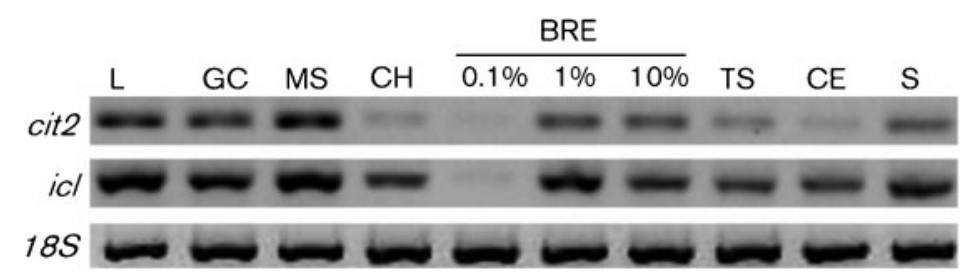

Fig. 5. Expression of Ma-cit2 and Ma-icl. RTPCR analysis from $M$. anisopliae grown in $1 \%$ locust cuticle (L), $1 \%$ giant cockroach cuticle (GC), $1 \%$ Manduca sexta cuticle (MS), $1 \%$ chitin $(\mathrm{CH}), 0.1 \%, 1 \%$ and $10 \%$ bean root exudate (BRE), 1\% tomato stem (TS), 1\% cellulose (CE) and $1 \%$ starch (S) grown for $12 \mathrm{~h}$. 

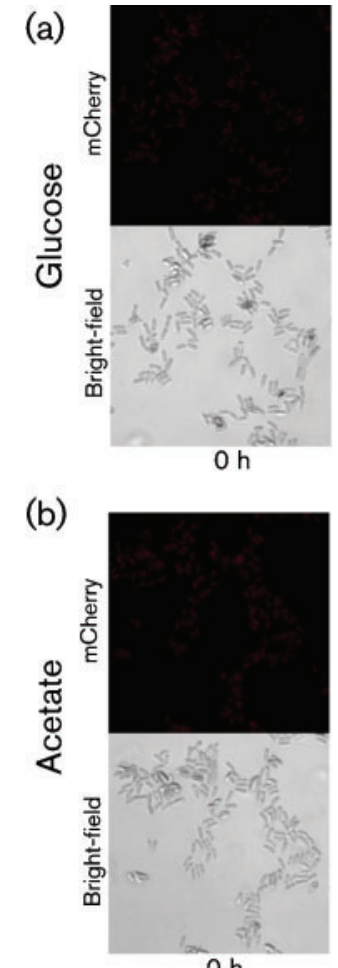

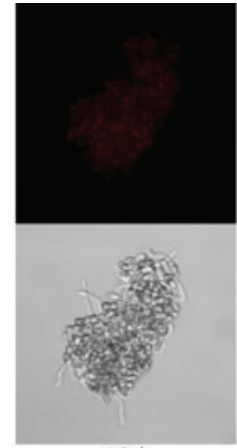

$12 \mathrm{~h}$

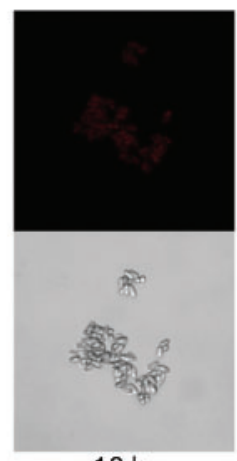

$12 \mathrm{~h}$

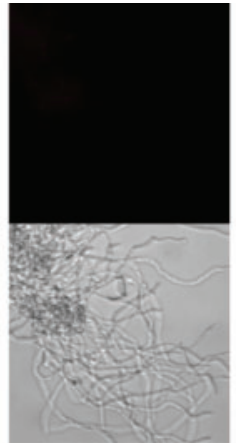

$24 \mathrm{~h}$

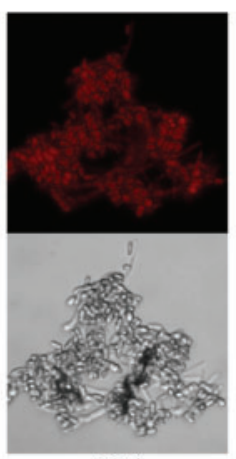

$24 \mathrm{~h}$

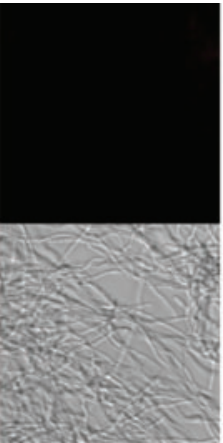

$36 \mathrm{~h}$

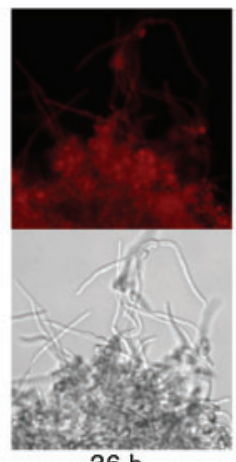

$36 \mathrm{~h}$

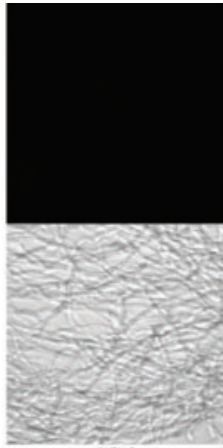

$48 \mathrm{~h}$

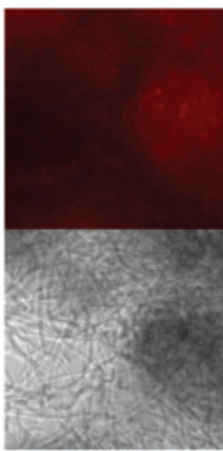

$48 \mathrm{~h}$

Fig. 6. Upregulation of $\mathrm{Ma}$-icl promoter. Time-course of pMa-Picl-mCherry construct expression in M. anisopliae grown in glucose $(a)$ or acetate $(b)$. Images were taken $(\times 40)$ by using light and fluorescence microscopy.

(a)

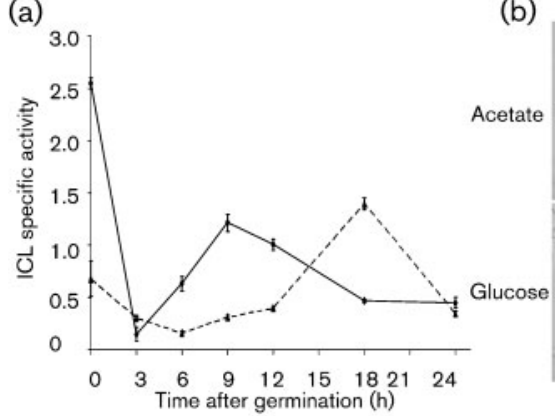

(c)

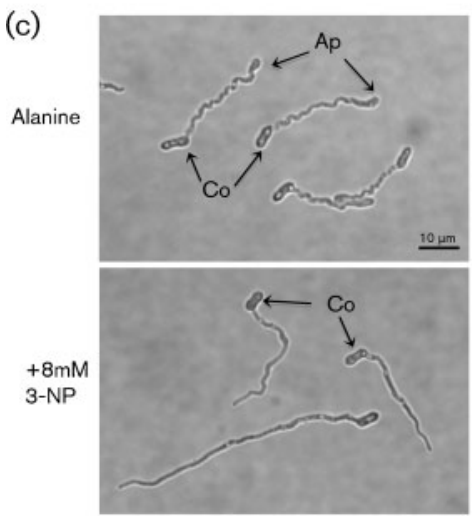

(b)

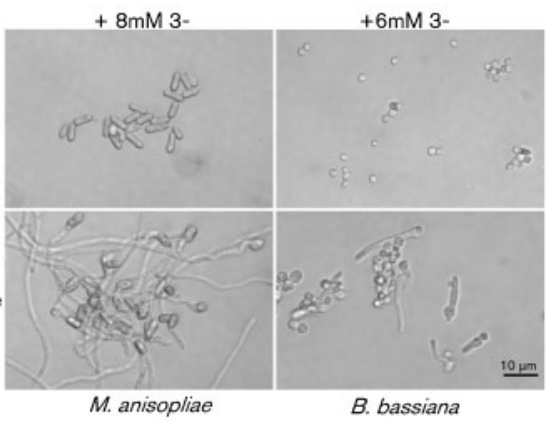

(d)

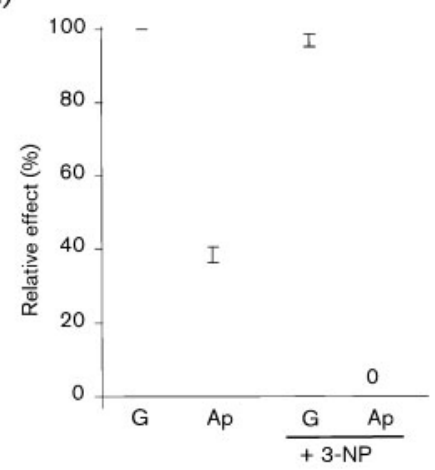

Fig. 7. $I C L$ activity in germinating conidia and effects of the ICL inhibitor 3-NP. (a) ICL specific activity during germination in glucose of $M$. anisopliae and B. bassiana. Specific activity is measured as $10^{-5} \times \mu \mathrm{mol}$ phenylhydrazine glyoxylate produced $\mathrm{min}^{-1}$ (mg protein $^{-1}$. (b) Conidial germination in $M$. anisopliae and $B$. bassiana on acetate and glucose medium with 3-NP added $24 \mathrm{~h}$ after inoculation. (c) Effect of 3-NP on appressorium (Ap) formation from germinated conidia $(\mathrm{Co})$ of M. anisopliae in $0.1 \%$ alanine. (d) Relative effect of 3-NP on germination (G) and appressorium (Ap) in M. anisopliae. (a) and (d) are means $\pm S D$ of three independent experiments. 


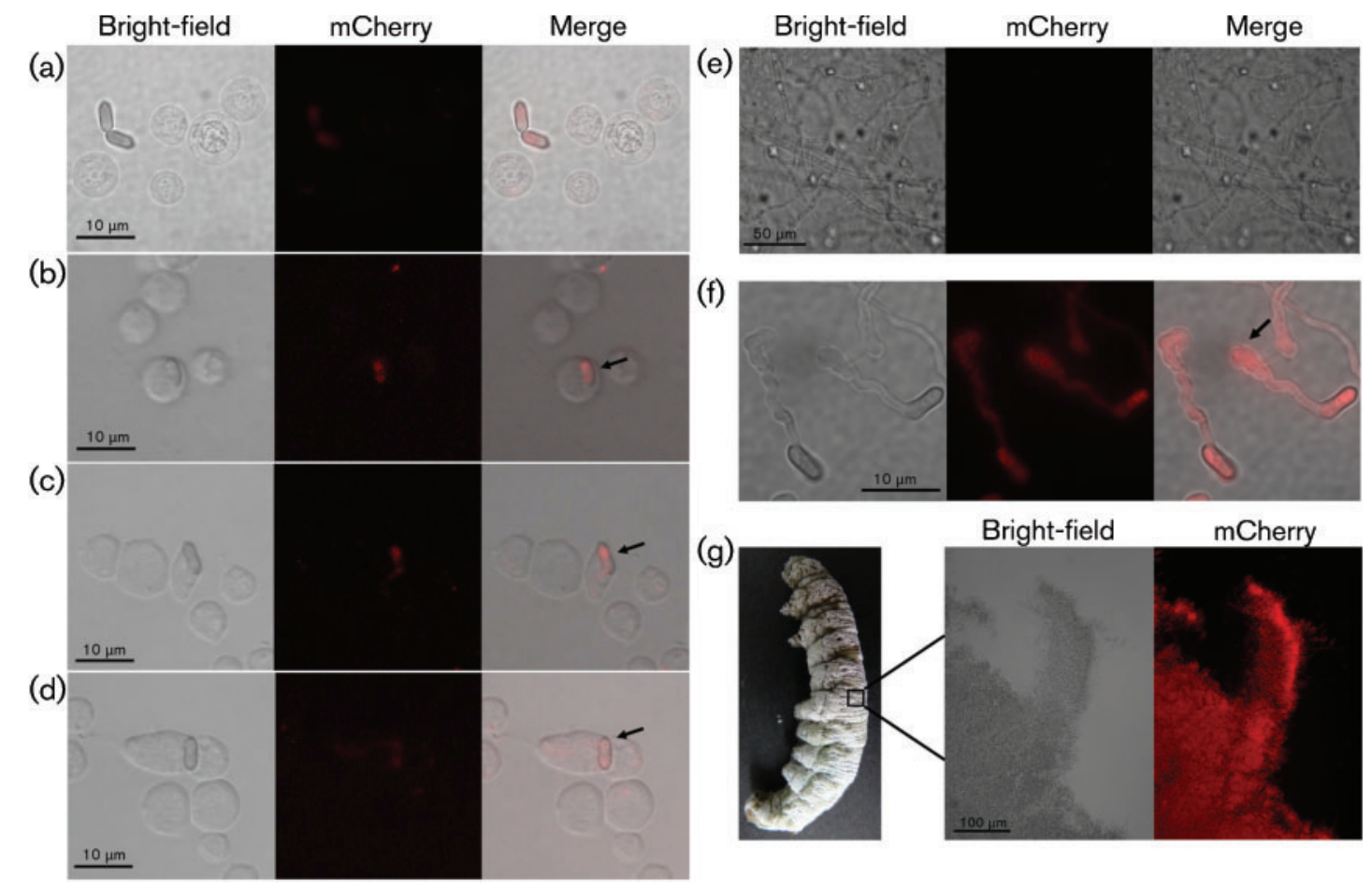

Fig. 8. Expression of $M$. anisopliae ICL (Ma-icl) in vivo. M. anisopliae was transformed with Ma-Picl-mCherry and expression was monitored $0 \mathrm{~h} \mathrm{(a),} 3 \mathrm{~h} \mathrm{(b),} 6 \mathrm{~h}$ (c), 9 h (d) and $48 \mathrm{~h}(\mathrm{e})$ post-infection in Manduca sexta. In (b)-(d), note that M. anisopliae has been phagocytosed by insect haemocytes and there is upregulation of the Ma-icl promoter (arrows). In (e), M. anisopliae mycelia have ramified in the haemocoel and $\mathrm{Ma}-\mathrm{icl}$ is not expressed. (f) Expression of $\mathrm{Ma}-\mathrm{ic} / 20 \mathrm{~h}$ after growth on $0.1 \%$ alanine on a Petri dish. Note the presence of appressorium (arrow). (g) Expression of Ma-icl 5 days post-infection as mycelia emerged from the insect cadaver.

pathogenic to humans or plants (Dunn et al., 2009). We demonstrated the enzymic presence of glyoxylate cycle intermediates in M. anisopliae and B. bassiana and confirmed this with RT-PCR. The presence of $\mathrm{C}_{2}$ compounds resulted in the upregulation of the glyoxylate cycle in these entomopathogenic fungi. The regulation of the glyoxylate cycle in $M$. anisopliae and B. bassiana was found to be comparable to those reported in other fungi, with respect to glucose and acetate catabolism (Bibbins et al., 1998; Chaure \& Connerton, 1995; De Lucas et al., 1994). In B. bassiana, the presence of glucose with acetate did not repress gene expression of the glyoxylate cycle genes, which has been observed in the fungus Leptosphaeria maculans (Idnurm \& Howlett, 2002).

One of the more interesting findings of this study was that the Ma-icl and Ma-cit2 genes share a bidirectional promoter. To our knowledge, this is first bidirectional promoter observed in M. anisopliae and the first bidirectional promoter involved in the regulation of glyoxylate cycle genes in any fungus. Bidirectional promoters have been described in other fungi for other genes, such as the DAC1-NAG1 promoter of C. albicans (Kumar et al., 2000), the GAL1-GAL10 promoter of Saccharomyces cerevisiae (Bash \& Lohr, 2001), and the niiA-niaD and prnD-prnB promoters of Aspergillus nidulans (Amaar \& Moore, 1998;
García et al., 2004). Studies of the human genome and the Arabidopsis thaliana genome show that genes with shared bidirectional promoters are often co-expressed and tend to be involved in similar biological functions (Trinklein et al., 2004; Wang et al., 2009). We also report two genes that encode citrate synthase, Ma-cit1 and Ma-cit2, which showed differential expression. In S. cerevisiae, three genes have been reported that code for this enzyme, induction of which was differentially induced by carbon source and different subcellular localization (Kim et al., 1986; Jia et al., 1997). In M. anisopliae, the bidirectional promoter $\mathrm{Ma}$-iclMa-cit2 and the citrate synthase genes may facilitate carbon flow between the TCA and the glyoxylate cycle.

We also investigated the contribution of the glyoxylate cycle in conidial germination and appressorium formation. We used acetate or glucose as a carbon source and the ICL inhibitor, 3 -NP, to show the utilization of the glyoxylate cycle in conidial germination. Germinating conidia of M. anisopliae and $B$. bassiana contained constitutive levels of ICL and germination was delayed in the presence of an ICL inhibitor, suggesting a role in conidial germination also described for Aspergillus fumigatus and Magnaporthe grisea (Ebel et al., 2006; Wang et al., 2003). The glyoxylate cycle plays a fundamental role in the utilization of $\mathrm{C}_{2}$ compounds, possibly in the form of 
storage lipids or fatty acids present in the conidia, which can be up to $48 \%$ of the conidial dry weight in Metarhizium (Pupin et al., 2000). The reserves of polyols and trehalose present in conidia of M. anisopliae and B. bassiana are not sufficient to generate the energy needed for germination (Hallsworth \& Magan, 1994). The glyoxylate cycle was upregulated during appressorium formation and during insect pathogenesis, but not during hyphal proliferation within the haemocoel. We detected the upregulation of $\mathrm{Ma}$-icl in the appressorium and confirmed the role of ICL by inhibiting appressorium formation using 3-NP. The role of the glyoxylate cycle in appressorium formation has been described in Magnaporthe grisea (Wang et al., 2003; Kim et al., 2006).

The human pathogen $C$. albicans can survive within mammalian macrophages, but icl mutants are unable to do so and are markedly less virulent in mice compared with wild-type strains (Lorenz \& Fink, 2001). Previously, we have shown that M. anisopliae can evade phagocytosis (Bidochka et al., 2010) and here we show that the glyoxylate cycle is upregulated when the fungus is within the insect haemocyte. Presumably, $M$. anisopliae is able to use $\mathrm{C}_{2}$ compounds from the hydrolysis of intracellular lipids within insect haemocytes. However, once the fungus evades phagocytosis and proliferates within the haemocoel, the glyoxylate cycle was repressed, presumably since readily metabolizable sugars, such as trehalose, are present. Once these sugars are depleted, the glyoxylate cycle is again upregulated in order to metabolize more complex carbon sources, such as lipids and fatty acids, during hyphal re-emergence from the insect cadaver, which is followed by conidiation.

The glyoxylate cycle allows for metabolic flexibility in $M$. anisopliae and B. bassiana and allows for the utilization of various carbon sources during insect pathogenesis. These findings suggest that the glyoxylate cycle could also be used during saprobic growth and/or plant rhizosphere colonization. An understanding of the contribution of the glyoxylate cycle in these fungi could allow for the development of strategies to generate hypervirulent strains. Particularly, more information on how the glyoxylate cycle interacts with fatty acid degradation pathways is needed. This could be of significant importance in elucidating the mechanisms and energetics of insect cuticular lipid degradation as well as haemocyte lipids, haemolymph and fat body utilization.

\section{ACKNOWLEDGEMENTS}

This research was conducted with the assistance of a Natural Sciences and Engineering Research Council of Canada Discovery Grant to M. J. B. We thank D. Gonzalez for enzyme assay experiments. I. E. P.-G. received a doctoral fellowship from Concejo National de Ciencia y Tecnología (Mexico).

\section{REFERENCES}

Amaar, Y. G. \& Moore, M. M. (1998). Mapping of the nitrateassimilation gene cluster ( $\mathrm{cr} A--n i i A-n i a D)$ and characterization of the nitrite reductase gene (niiA) in the opportunistic fungal pathogen Aspergillus fumigatus. Curr Genet 33, 206-215.

Araujo-Palomares, C. L., Riquelme, M. \& Castro-Longoria, E. (2009). The polarisome component SPA-2 localizes at the apex of Neurospora crassa and partially colocalizes with the Spitzenkorper. Fungal Genet Biol 46, 551-563.

Arrese, E. L. \& Soulages, J. L. (2010). Insect fat body: energy, metabolism, and regulation. Annu Rev Entomol 55, 207-225.

Bash, R. \& Lohr, D. (2001). Yeast chromatin structure and regulation of GAL gene expression. Prog Nucleic Acid Res Mol Biol 65, 197-259.

Bibbins, M., Sheffield, P. J., Gainey, L. D., Mizote, T. \& Connerton, I. F. (1998). Promoter analysis of the acetate-inducible isocitrate lyase gene (acu-3) from Neurospora crassa. Biochim Biophys Acta 1442, 320325.

Bidochka, M. J., Clark, D. C., Lewis, M. \& Keyhani, N. O. (2010). Could insect phagocytic avoidance by entomogenous fungi have evolved via selection against soil amoeboid predators? Microbiology 156, 2164-2171.

Chaure, P. T. \& Connerton, I. F. (1995). Derepression of the glyoxylate cycle in mutants of Neurospora crassa accelerated for growth on acetate. Microbiology 141, 1315-1320.

Cho, E. M., Boucias, D. \& Keyhani, N. O. (2006a). EST analysis of cDNA libraries from the entomopathogenic fungus Beauveria (Cordyceps) bassiana. II. Fungal cells sporulating on chitin and producing oosporein. Microbiology 152, 2855-2864.

Cho, E. M., Liu, L., Farmerie, W. \& Keyhani, N. O. (2006b). EST analysis of cDNA libraries from the entomopathogenic fungus Beauveria (Cordyceps) bassiana. I. Evidence for stage-specific gene expression in aerial conidia, in vitro blastospores and submerged conidia. Microbiology 152, 2843-2854.

Clarkson, J. M. \& Charnley, A. K. (1996). New insights into the mechanisms of fungal pathogenesis in insects. Trends Microbiol 4, 197-203.

Cubero, B. \& Scazzocchio, C. (1994). Two different, adjacent and divergent zinc finger binding sites are necessary for CREA-mediated carbon catabolite repression in the proline gene cluster of Aspergillus nidulans. EMBO J 13, 407-415.

De Lucas, J. R., Gregory, S. \& Turner, G. (1994). Analysis of the regulation of the Aspergillus nidulans acuD gene, encoding isocitrate lyase, by construction of a hybrid promoter. Mol Gen Genet 243, 654659.

Dunn, M. F., Ramirez-Trujillo, J. A. \& Hernandez-Lucas, I. (2009). Major roles of isocitrate lyase and malate synthase in bacterial and fungal pathogenesis. Microbiology 155, 3166-3175.

Ebel, F., Schwienbacher, M., Beyer, J., Heesemann, J., Brakhage, A. A. \& Brock, M. (2006). Analysis of the regulation, expression, and localisation of the isocitrate lyase from Aspergillus fumigatus, a potential target for antifungal drug development. Fungal Genet Biol 43, 476-489.

Fang, W., Pei, Y. \& Bidochka, M. J. (2006). Transformation of Metarhizium anisopliae mediated by Agrobacterium tumefaciens. Can J Microbiol 52, 623-626.

Fang, W., Pei, Y. \& Bidochka, M. J. (2007). A regulator of a G protein signalling (RGS) gene, cag8, from the insect-pathogenic fungus Metarhizium anisopliae is involved in conidiation, virulence and hydrophobin synthesis. Microbiology 153, 1017-1025.

García, I., Gonzalez, R., Gomez, D. \& Scazzocchio, C. (2004). Chromatin rearrangements in the prnD-prnB bidirectional promoter: dependence on transcription factors. Eukaryot Cell 3, 144-156.

Hallsworth, J. E. \& Magan, N. (1994). Effect of carbohydrate type and concentration on polyhydroxy alcohol and trehalose content of 
conidia of three entomopathogenic fungi. Microbiology 140, 27052713.

Hu, G. \& St Leger, R. J. (2002). Field studies using a recombinant mycoinsecticide (Metarhizium anisopliae) reveal that it is rhizosphere competent. Appl Environ Microbiol 68, 6383-6387.

Idnurm, A. \& Howlett, B. J. (2002). Isocitrate lyase is essential for pathogenicity of the fungus Leptosphaeria maculans to canola (Brassica napus). Eukaryot Cell 1, 719-724.

Jarrold, S. L., Moore, D., Potter, U. \& Charnley, A. K. (2007). The contribution of surface waxes to pre-penetration growth of an entomopathogenic fungus on host cuticle. Mycol Res 111, 240-249.

Jia, Y. K., Bécam, A. M. \& Herbert, C. J. (1997). The CIT3 gene of Saccharomyces cerevisiae encodes a second mitochondrial isoform of citrate synthase. Mol Microbiol 24, 53-59.

Kim, K. S., Rosenkrantz, M. S. \& Guarente, L. (1986). Saccharomyces cerevisiae contains two functional citrate synthase genes. Mol Cell Biol 6, 1936-1942.

Kim, S.-Y., Park, J.-S. \& Oh, K.-B. (2006). Effects of isocitrate lyase inhibitors on spore germination and appressorium development in Magnaporthe grisea. J Microbiol Biotechnol 16, 1158-1162.

Kumar, M. J., Jamaluddin, M. S., Natarajan, K., Kaur, D. \& Datta, A. (2000). The inducible $N$-acetylglucosamine catabolic pathway gene cluster in Candida albicans: discrete $\mathrm{N}$-acetylglucosamine-inducible factors interact at the promoter of NAG1. Proc Natl Acad Sci U S A 97, 14218-14223.

Lorenz, M. C. \& Fink, G. R. (2001). The glyoxylate cycle is required for fungal virulence. Nature 412, 83-86.

Prasad, S. V., Ryan, R. O., Law, J. H. \& Wells, M. A. (1986). Changes in lipoprotein composition during larval-pupal metamorphosis of an insect, Manduca sexta. J Biol Chem 261, 558-562.

Pupin, A. M., Messias, C. L., Piedrabuena, A. E. \& Roberts, D. W. (2000). Total lipids and fatty acids of strains of Metarhizium anisopliae. Braz J Microbiol 31, 121-128.

Ravagnani, A., Gorfinkiel, L., Langdon, T., Diallinas, G., Adjadj, E., Demais, S., Gorton, D., Arst, H. N., Jr \& Scazzocchio, C. (1997). Subtle hydrophobic interactions between the seventh residue of the zinc finger loop and the first base of an HGATAR sequence determine promoter-specific recognition by the Aspergillus nidulans GATA factor AreA. EMBO J 16, 3974-3986.

Rose, T. M., Henikoff, J. G. \& Henikoff, S. (2003). CODEHOP (COnsensus-DEgenerate Hybrid Oligonucleotide Primer) PCR primer design. Nucleic Acids Res 31, 3763-3766.
Schloss, J. V. \& Cleland, W. W. (1982). Inhibition of isocitrate lyase by 3 -nitropropionate, a reaction-intermediate analogue. Biochemistry 21, 4420-4427.

Small, C. L. \& Bidochka, M. J. (2005). Up-regulation of Prl, a subtilisin-like protease, during conidiation in the insect pathogen Metarhizium anisopliae. Mycol Res 109, 307-313.

St Leger, R. J. (2008). Studies on adaptations of Metarhizium anisopliae to life in the soil. J Invertebr Pathol 98, 271-276.

St Leger, R. J., Butt, T. M., Goettel, M. S., Staple, R. C. \& Roberts, D. W. (1989). Production in vitro of appressoria by the entomopathogenic fungus Metarhizium anisopliae. Exp Mycol 13, 274-288.

Tan, G., Gao, Y., Shi, M., Zhang, X., He, S., Chen, Z. \& An, C. (2005). SiteFinding-PCR: a simple and efficient PCR method for chromosome walking. Nucleic Acids Res 33, e122.

Thomas, M. B. \& Read, A. F. (2007). Can fungal biopesticides control malaria? Nat Rev Microbiol 5, 377-383.

Todd, R. B., Andrianopoulos, A., Davis, M. A. \& Hynes, M. J. (1998). $\mathrm{FacB}$, the Aspergillus nidulans activator of acetate utilization genes, binds dissimilar DNA sequences. EMBO J 17, 2042-2054.

Trinklein, N. D., Aldred, S. F., Hartman, S. J., Schroeder, D. I., Otillar, R. P. \& Myers, R. M. (2004). An abundance of bidirectional promoters in the human genome. Genome Res 14, 62-66.

Wang, C. \& St Leger, R. J. (2006). A collagenous protective coat enables Metarhizium anisopliae to evade insect immune responses. Proc Natl Acad Sci U S A 103, 6647-6652.

Wang, C. \& St Leger, R. J. (2007). The MAD1 adhesin of Metarhizium anisopliae links adhesion with blastospore production and virulence to insects, and the MAD2 adhesin enables attachment to plants. Eukaryot Cell 6, 808-816.

Wang, Z. Y., Thornton, C. R., Kershaw, M. J., Debao, L. \& Talbot, N. J. (2003). The glyoxylate cycle is required for temporal regulation of virulence by the plant pathogenic fungus Magnaporthe grisea. Mol Microbiol 47, 1601-1612.

Wang, C., Duan, Z. \& St Leger, R. J. (2008). MOS1 osmosensor of Metarhizium anisopliae is required for adaptation to insect host hemolymph. Eukaryot Cell 7, 302-309.

Wang, Q., Wan, L., Li, D., Zhu, L., Qian, M. \& Deng, M. (2009). Searching for bidirectional promoters in Arabidopsis thaliana. BMC Bioinformatics 10 (Suppl. 1), S29.

Edited by: B. A. Horwitz 